\title{
Descripción de los cubículos utilizados en granjas lecheras en el sur de Chile y su relación con el confort de las vacas ${ }^{\#}$
}

\author{
Free stall design and dimensions on dairy farms in southern Chile, \\ and its relationship with cow comfort
}

\author{
JD Córdoba ${ }^{a}$, MP Castillo ${ }^{b}, \mathbf{N}$ Ormeño ${ }^{b}$, G Acosta $^{\mathrm{c}}, \mathbf{N ~ T a d i c h}^{\mathrm{b} *}$ \\ ${ }^{a}$ Magíster en Ciencias mención Salud Animal, Universidad Austral de Chile, Valdivia, Chile. \\ bInstituto de Ciencias Clínicas Veterinarias, Facultad de Ciencias Veterinarias, Universidad Austral de Chile, Valdivia, Chile. \\ 'Instituto de Medicina Preventiva Veterinaria, Facultad de Ciencias Veterinarias, Universidad Austral de Chile, Valdivia, Chile.
}

\section{SUMMARY}

\begin{abstract}
Uncomfortable stalls and beds reduce the time that cows spend resting and are a risk factor for lameness. In Chile there is little information about the dimensions and bed stalls used for dairy cows. The aim of this study was to determine the characteristics of stalls and beds used for dairy cows in southern Chile. Fifty dairy farms with more than 100 lactating dairy cows were visited. Dimensions and bedding of 1,068 (10\%) of all cubicles available, was recorded. Records were entered into an Excel spreadsheet and analyzed using descriptive statistics. Results are presented as percentages, means, ranges and medians. An 84\% (42) of the farms housed their cows for five months during winter; an $45.5 \%$ (29) of them had more than one stall/cow and 34\% (15) had less than one stall/cow. The most frequently used stall-bedding combination was metal stall with mattresses filled with rubber or soil bedding, and wooden stall/soil bedding. More than $70 \%$ of cubicles were below the reference values for length of the stall, height of neck rail, height to upper stall division and distance from the wall to neck rail; $45 \%$ of stalls had values below the recommended guidelines for the lower stall division. Height of the curb and brisket board exceeded the recommendations in more than $20 \%$ of stalls. Dairy farms used mainly night confinement in winter and early spring months. Stalls most commonly used were metallic, North American model. Their dimensions and bedding do not comply with the recommendations of the literature.
\end{abstract}

Palabras claves: cubículos, confort vacas, bienestar.

Key words: free stalls, cow comfort, welfare.

\section{INTRODUCCIÓN}

La producción lechera en el sur de Chile está basada principalmente en sistemas pastoriles (Balocchi y col 2002). Sin embargo, por su clima con alta pluviometría en invierno, se presta para utilizar sistemas de confinamiento. Trabajos anteriores indican que no más del $42 \%$ de las lecherías estabulan las vacas total o parcialmente durante el invierno (Ernst y col 2000). Los sistemas de estabulación deben ofrecer a las vacas comodidades para no afectar su bienestar, entre ellas, los cubículos y las camas deben ser lo más confortables posible para evitar problemas de cojeras y otras enfermedades como mastitis (Cook y Nordlund 2009, Bernardi y col 2009). Entre los diseños de cubículos para las vacas estabuladas, los cubículos libres o free stalls son los que han adquirido más popularidad, entre otras cosas, por el aprovechamiento del espacio, la facilidad de limpieza y la facilidad de circulación de las vacas dentro del sistema. Sin embargo, si los cubículos están mal diseñados pueden producir problemas tales como

Aceptado: 06.10.2011.

\#Proyecto FONDECYT 1090373

* Casilla Postal 567, Valdivia, Chile; ntadich@uach.cl vacas acostadas en los pasillos o en áreas de ejercicio, haciendo que sean susceptibles a lesiones producidas por otras vacas o a cojeras (Veissier y col 2004) o extender el tiempo en que las vacas permanecen de pie, lo cual induce a la presentación de diferentes lesiones del tejido córneo del pie (Vokey y col 2001). Los cambios conductuales que adquieren las vacas estabuladas producto de un mal diseño de los cubículos han sido bien documentados ${ }^{1}$. Considerando el bienestar del animal, independientemente del sistema de estabulación y manejo utilizado, la vaca debe ser tratada con cuidado y debe ser provista con un espacio confortable para descansar y tener un fácil acceso al agua y comida (Cook y Nordlund 2009).

El tema del bienestar de la vaca ha recibido considerable interés durante la década pasada. Cubículos no adecuados reducen el tiempo de reposo de la vaca y son un riesgo para la presentación de cojeras (Espejo y Endres 2007). En Chile existe escasa literatura respecto a las características de los sistemas utilizados para estabular las vacas lecheras (Tadich y Ormeño 2004). Basado en trabajos anteriores de estos autores, la hipótesis planteada fue que las condiciones

\footnotetext{
Anderson N. 2003. Free stall dimensions. Livestock Technology. Ministry of Agriculture and Food and Rural Affairs. www.omafra. gov.on.ca. Northern Ontario Regional Office: OMAF, Canada.
} 
de estabulación ofrecidas a las vacas lecheras en el sur de Chile no están de acuerdo a las recomendaciones entregadas en la literatura internacional. El presente trabajo entrega información acerca de las dimensiones, material de construcción de los cubículos y camas empleadas para la estabulación de vacas lecheras en 50 lecherías del sur de Chile y analiza los problemas de bienestar que se pueden producir cuando no se cumplen las recomendaciones entregadas en la literatura.

\section{MATERIAL Y MÉTODOS}

Entre junio y noviembre de 2009, se visitaron 50 lecherías ubicadas en las regiones de La Araucanía, de Los Lagos y de Los Ríos, Chile. Las lecherías fueron contactadas por medio de industrias y compañías comerciales vinculadas al sector lechero, como también a través de los médicos veterinarios y/o propietarios de las lecherías. Se seleccionaron aquellas que tenían más de 100 vacas en producción, cuyo establo estuviera o hubiera sido utilizado durante el período del estudio, y por la disposición de los dueños a participar.

Las lecherías se visitaron por una sola vez. En cada visita se aplicó una encuesta mediante la cual se obtuvieron antecedentes acerca del período de estabulación, el número de meses que se estabulaba y el número de horas del día en que las vacas estaban estabuladas. Durante la visita se escogió al azar el 10\% de los cubículos en distintos sectores del establo, separados uno del otro por un intervalo de 6 a 8 cubículos y se registraron sus medidas en una planilla previamente diseñada para este efecto. En relación a los cubículos se recolectaron los siguientes antecedentes:

Cuadro 1. Distribución porcentual de las medidas obtenidas de 1.068 cubículos, en relación a los rangos de referencia, en 50 lecherías del sur de Chile. southern Chile.

Proportional distribution of the measures obtained from 1,068 cubicles, in relationship to the range of reference, on 50 dairy farms of

\begin{tabular}{|c|c|c|c|c|c|c|c|}
\hline & $\begin{array}{l}\text { Valor } \\
\text { referencia* } \\
\quad(\mathrm{cm})\end{array}$ & Media \pm DE & $\begin{array}{l}\mathrm{N}^{\mathrm{o}} \text { y } \% \text { de } \\
\text { cubículos } \\
\text { menores al } \\
\text { límite inferior } \\
\text { de referencia }\end{array}$ & $\begin{array}{l}\mathrm{N}^{\mathrm{o}} \text { y } \% \text { de } \\
\text { cubículos } \\
\text { entre el rango } \\
\text { de referencia }\end{array}$ & $\begin{array}{c}\mathrm{N}^{\mathrm{o}} \text { y } \% \text { de } \\
\text { cubículos } \\
\text { mayores al } \\
\text { límite superior } \\
\text { de referencia }\end{array}$ & Moda & Rango \\
\hline $\begin{array}{l}\text { Distancia del } \\
\text { muro al riel del } \\
\text { cuello }(\mathrm{cm})\end{array}$ & $\begin{array}{l}\geq 69 \\
66\end{array}$ & $56,25 \pm 12,77$ & $825(83,5 \%)$ & $164(16,5 \%)$ & - & 50 & 15 a 96 \\
\hline $\begin{array}{l}\text { Alto de la } \\
\text { solera } \\
(\mathrm{cm})\end{array}$ & $15-25$ & $21,27 \pm 4,89$ & $121(11,4 \%)$ & $721(67,9 \%)$ & $220(20,7 \%)$ & 20 & 8 a 36 \\
\hline $\begin{array}{l}\text { Alto riel } \\
\text { del cuello } \\
(\mathrm{cm})\end{array}$ & $122-127$ & $105,77 \pm 10,07$ & $716(72,5 \%)$ & $220(22,3 \%)$ & $51(5,2 \%)$ & 103 & 69 a 138 \\
\hline $\begin{array}{l}\text { Largo del } \\
\text { cubículo } \\
(\mathrm{cm})\end{array}$ & $243-273$ & $223,8 \pm 12,36$ & $999(93,7 \%)$ & $66(6,2 \%)$ & $1(0,1 \%)$ & 225 & 187 a 313 \\
\hline $\begin{array}{l}\text { Alto al riel } \\
\text { divisor inferior } \\
(\mathrm{cm})\end{array}$ & $28-30$ & $31,74 \pm 15,9$ & $474(45,1 \%)$ & $307(29,2 \%)$ & $269(25,7 \%)$ & 20 & 0 a 95 \\
\hline $\begin{array}{l}\text { Alto al riel } \\
\text { divisor superior } \\
(\mathrm{cm})\end{array}$ & $122-127$ & $97,36 \pm 10,98$ & $847(79,4 \%)$ & $75(7 \%)$ & $144(13,6 \%)$ & 102 & 56 a 137 \\
\hline $\begin{array}{l}\text { Ancho } \\
\text { cubículo } \\
(\mathrm{cm})\end{array}$ & $117-132$ & $115,29 \pm 21,2$ & $291(27,3 \%)$ & $687(64,4 \%)$ & $88(8,3 \%)$ & 114 & 63 a 238 \\
\hline $\begin{array}{l}\text { Largo del riel del } \\
\text { cuello a la solera } \\
(\mathrm{cm})\end{array}$ & $\begin{array}{c}162-178 \\
183\end{array}$ & $167,85 \pm 12,83$ & $281(28,3 \%)$ & $379(38,2 \%)$ & $330(33,5 \%)$ & 170 & 106 a 222 \\
\hline $\begin{array}{l}\text { Altura del } \\
\text { posicionador del } \\
\text { pecho }(\mathrm{cm})\end{array}$ & $10-15$ & $19,23 \pm 7,17$ & - & $155(64 \%)$ & $87(36 \%)$ & 12 & 4 a 42 \\
\hline
\end{tabular}


número total de cubículos, número de cubículos por animal, largo total del cubículo, distancia desde el muro al riel del cuello, largo desde el riel del cuello a la solera, ancho del cubículo, alto al riel del cuello, alto al riel divisorio inferior y superior, espacio entre riel divisorio inferior y superior, alto de la solera y alto del contenedor del pecho y largo del riel divisorio. En lo que respecta a la información de las camas se consideró el material de la cama, la cantidad de ella y su estado; además se consultó sobre la frecuencia de limpieza y cambio de la cama. El diseño de los cubículos se realizó en base a la descripción entregada por Veissier y col (2004).

Los antecedentes recopilados fueron introducidos en una planilla EXCEL y se presentan en forma de estadística descriptiva expresada en cuadros con base en porcentajes, promedios, rangos, medias y moda. Los valores referenciales con los cuales se compararon los obtenidos en este trabajo fueron obtenidos de los trabajos de Anderson ${ }^{2}$ y Palmer (2005), para vacas raza Holstein Friesian y Frisón, entre 500 a $700 \mathrm{~kg}$ de peso vivo.

\section{RESULTADOS Y DISCUSIÓN}

Los rebaños tenían un promedio de $315 \pm 151$ vacas (rango 120 - 830), con una producción promedio de leche de $22 \pm 5$ L/día (rango12 a 40 L/día). En un $88 \%$ de las lecherías las vacas eran de la raza Holstein Friesian (66\%) o Frisón Negro (22\%), las otras lecherías tenían Montbeliarde (8\%) el otro 4\% tenían Frisón Rojo, Ayrshire x Rojo Sueco, en distintas proporciones.

Un 84\% (42) de las lecherías visitadas estabulaban de forma estacional, siendo cinco meses (mayo a septiembre) el período más frecuente de estabulación (28\%) y un $16 \%$ (8) lo hacían en forma permanente. Un 62\% estabulaban sólo durante la noche. En las ocho lecherías con estabulación anual, sólo una (12,5\%) estabulaba únicamente en la noche. El tiempo de estabulación fue menor al informado por Tadich y col (1998) y Tadich y Ormeño (2004), en lecherías de las Regiones de La Araucanía y de Los Lagos.

El 88\% ( $n=44)$ de las lecherías tenían cubículos libres y un $10 \%(\mathrm{n}=5)$ utilizaban cama caliente. Una de las lecherías (2\%) que estabulaba en cama caliente (opción incluida en el grupo "No aplica") tenía un galpón con cubículos de metal de uso intermitente. El porcentaje de las lecherías que utilizaban cubículos y estabulaban las vacas durante la noche concuerda con Tadich y Ormeño (2004). Blowey y Edmondson (2000) señalan que los ganaderos prefieren utilizar este tipo de cubículos, ya que cada vaca puede disfrutar de un espacio individual para su descanso.

De aquéllas que utilizaban cubículos un $45 \%$ (20) tenían más de uno por vaca estabulada (rango:1,2-4,4) y un $34 \%$ (15) tenían menos de uno por vaca estabulada.

Anderson N. 2007, Cow behaviour to judge free stall and tie stall barns. Livestock Technology. INFOSheet, Ministry of Agriculture, Food and Rural Affairs. www.omafra.gov.on.ca. Ontario, Canada.
Que un 34\% de las lecherías visitadas tuvieran menos de un cubículo por animal es un problema, ya que deberían existir suficientes cubículos para todos los animales estabulados, evitando el rechazo y la competencia por ellos y que algunas de las vacas se acuesten en el pasillo (von Keyserlingk y Weary 2009). La escasez de cubículos se asocia con traumas y lesiones (Wathes y Charles 1994). Algunos autores recomiendan 90 cubículos/100 animales (Radostits y col 1994, Wathes y Charles 1994). Sin embargo, esto disminuye los tiempos de descanso de las vacas, aumentando la permanencia de pie, con un aumento de las lesiones del tejido córneo de las pezuñas (Wierenga y Hopster 1990, Webster 2002).

En el 55,6\% (25) de las lecherías que utilizaban cubículos éstos eran sólo metálicos, en un $20 \%$ (9) sólo de madera y en un $24,4 \%$ (11) de ambos materiales. El diseño norteamericano metálico (arriñonado) fue el más utilizado, seguido por el cubículo de madera. Los cubículos metálicos con diseño norteamericano tienen la ventaja que permiten que la vaca se acueste de forma diagonal y pueda introducir la cabeza en los cubículos adyacentes, ampliando su espacio de descanso. Un mal diseño puede resultar en traumas (McFarland 2000, Weschsler y col 2000), reducción en el tiempo de descanso e incremento del tiempo de pie sobre los pasillos (Nordlund y Cook 2003), existiendo una relación entre el aumento de horas de pie sobre el concreto y la presentación de laminitis (Leonard y col 1994, 1996, Chaplin y col 2000).

Se consideraron las recomendaciones más recientes para el largo total de la cama del cubículo, las que están en un rango de 240 a $274 \mathrm{~cm}$ (Hughes 2000, Anderson 2003, Palmer 2005). Sólo un 6,2\% de los cubículos cumplía con la longitud recomendada y un $93,7 \%$ de los cubículos tenían longitudes inferiores. Faull y col (1996), en Inglaterra, encontraron que el $87 \%$ de los cubículos examinados medía menos de $230 \mathrm{~cm}$ de largo. Los cubículos cortos producen diversos problemas, exposición de la parte posterior de la vaca hacia el pasillo, exponiendo los miembros y la ubre a traumatismos, rechazo de la vaca a entrar al cubículo, manteniéndose parada con los miembros anteriores en la cama y los posteriores en el pasillo, "perching". Esta postura ha demostrado aumentar las lesiones podales y las cojeras (Flower y Weary 2002, Bernardi y col 2009). La gran cantidad de cubículos que no cumplían con el largo adecuado puede deberse a una obsolescencia de éstos, a la fuerte inclusión de genética Holstein en los rebaños, o a la falta de asesoría al momento de construir los galpones y cubículos.

Las recomendaciones para el ancho del cubículo, generalmente, se dan en términos del tamaño del animal. La recomendación general es que el ancho del cubículo debe ser el doble del ancho de la cadera, lo que significa aproximadamente 100 a $120 \mathrm{~cm}$ (Irish y Martin 1983, McFarland y Gamroth 1994). Cuando la vaca se acuesta, las vísceras abdominales se desplazan distendiendo el abdomen y aumentando el espacio que ocupa en el cubículo; 
si la vaca no está cómoda rehusará utilizar el cubículo y preferirá acostarse en los pasillos. Esto se agrava cuando el riel inferior es de metal o madera, lo que impide su desplazamiento, comprimiendo las vísceras (Hughes 2000). En este estudio se utilizó un rango de 117 a $132 \mathrm{~cm}$ de ancho; sin embargo, un $77 \%$ de los cubículos presentaron anchos inferiores a los valores de referencia.

El posicionador del pecho limita el espacio real con que cuenta la vaca para permanecer echada y generalmente se ubica a 168-182 cm del bordillo (Palmer 2005). La altura del posicionador es un factor importante en la comodidad de la vaca, ya que cuando es muy alto o de bordes agudos impide la correcta extensión de la mano al pararse (Nordlund y Cook 2003, Palmer 2005). Un 64\% de los cubículos que contaban con posicionadores del pecho tenían una altura superior a la recomendada. Se ha demostrado una asociación significativa entre la altura del posicionador y la presencia de cojeras, por lo que se recomienda que ésta no supere los $15 \mathrm{~cm}$ y que preferentemente sea de $10 \mathrm{~cm}$ para permitir un adecuado estiramiento y un levantarse más natural en la vaca (Nordlund y Cook 2003). Palmer (2005) y Espejo y Endres (2007) indican que cuando el posicionador es más alto de $15 \mathrm{~cm}$ y el área entre él y el muro u otro cubículo está rellena con concreto, aumenta la prevalencia de cojeras.

En un 20,7\% de los cubículos la altura de la solera fue superior a la recomendada. La altura excesiva de la solera produce rechazo de la vaca por el cubículo, debido a las dificultades de la salida de la vaca del cubículo, produciendo una carga excesiva del peso sobre los miembros posteriores y tendones flexores (Hughes 2000). Nordlund y Cook (2003) señalan que en Norteamérica las alturas de las soleras son mayores a las recomendadas en Europa, variando entre 20 y $35 \mathrm{~cm}$, para evitar ensuciar las camas cuando se lavan y limpian los pasillos.

El riel del cuello tiene un papel muy importante para regular el mayor o menor uso de la cama por parte de la vaca antes de echarse. Rieles del cuello en posiciones muy posteriores hacen que la vaca rechace el cubículo o que al echarse quede con parte del cuerpo fuera de él. En este estudio un 28,3\% de los cubículos presentó rieles del cuello posicionados muy atrás, lo que sugiere que en estas lecherías pudiera existir una disminución de los tiempos de descanso de las vacas. Por otra parte, los rieles muy anteriores permiten que la vaca defeque y orine en la cama al pararse (Anderson 2007). Bernardi y col (2009) indican que cubículos sin rieles de cuello presentaron 9 veces más contaminación que aquéllos con presencia del riel del cuello. La posición del riel del cuello es fácil de cambiar y se refleja en una mayor comodidad para la vaca y mayor utilización del cubículo. Tucker y col (2005) señalan que cuando el riel está muy bajo o muy cerca de la solera las vacas permanecen menos tiempo paradas en el cubículo. Fulwider y Palmer (2004) indican que el porcentaje de tiempo que las vacas permanecen acostadas en cubículos con cama de colchoneta se incrementa cuando la altura del riel del cuello aumenta de 114 a $127 \mathrm{~cm}$. En este estudio, un 72,5\% de los cubículos medidos tuvieron una altura inferior a la recomendada, lo cual podría afectar los tiempos de descanso.

La vaca al pararse necesita un espacio adicional para llevar a cabo estos movimientos en la forma más natural posible. Ceballos y col (2004) indican que entre el riel del cuello y el muro o el cubículo opuesto, las vacas ocupan un espacio de $60 \mathrm{~cm}$ a un máximo de $80 \mathrm{~cm}$. En este estudio utilizamos como valor mínimo $66 \mathrm{~cm}$, por lo que un $83,5 \%$ de los cubículos no cumplieron con la distancia requerida. La dificultad para pararse produce un rechazo de la vaca a ocupar el cubículo y disminuye los tiempos de descanso.

La altura de los rieles divisorios debe ser determinada de acuerdo a la altura en la cual se producen los máximos desplazamientos laterales de la cadera de la vaca. Ceballos y col (2004) encontraron dos zonas donde esto ocurre: una alta, a $95-135 \mathrm{~cm}$ sobre el piso, y una baja a $10-50 \mathrm{~cm}$ del piso. Ellos sugieren que las barras divisoras inferior y superior deben ubicarse a $70 \mathrm{~cm}$ y $150 \mathrm{~cm}$ del piso, respectivamente, lo que evitará que las caderas de las vacas tomen contacto con ellas y se produzcan lesiones. En nuestro estudio se utilizaron valores referenciales para el riel superior, inferiores a los entregados por estos autores; a pesar de esto, un 79,4\% tuvo valores menores a los recomendados. Para el riel inferior, un $45,1 \%$ estuvo bajo los valores de referencia. La falta de una altura adecuada del riel inferior puede producir entrampamiento de las extremidades bajo el riel y en el caso del riel superior, golpes en los huesos de las caderas, al momento de echarse o pararse las vacas.

En cuanto al tipo de cama y su combinación con el tipo de cubículo se observó que las lecherías presentaban más de una combinación de cubículo y camas. La más utilizada fueron los cubículos metálicos norteamericanos con cama de colchón relleno con caucho (28\%), cubículos metálicos con cama de tierra (22\%) o cubículos de madera con cama de tierra (22\%). Un $43 \%$ (20) de las lecherías visitadas tenían camas en base a tierra, la cual no es considerada como una de las mejores (Hughes 2000). La tierra para ser utilizada como cama debe ser seleccionada, sin piedras $\mathrm{u}$ otros materiales que pueden causar heridas e incomodidad al animal; una de sus desventajas es que requiere mayor mantenimiento (Faull y Hughes 1985). Un 46\% (21) de las lecherías usaban colchón relleno de caucho picado o paja. Este tipo de cama se endurece y adquiere la forma del animal, desplazándose el relleno hacia los lados, por lo que con el tiempo y poco mantenimiento la cama se torna cada vez más incómoda.

En nuestro estudio sólo un 9\% (4) de las lecherías utilizaban arena como cama. La arena es el material óptimo (Cook 2009), pues no permite la multiplicación de microorganismos. La complicación de su uso está en su decantación en los pozos purineros (Callejo y Jimeno 1998). Drissler y col (2005) señalan que el tiempo de reposo de las vacas está asociado a los niveles de arena 
de las camas; por cada centímetro de disminución de la cama de arena, las vacas permanecieron 11 minutos menos echadas. La arena provee a la vaca de amortiguación, tracción y soporte, lo que facilita los movimientos de pararse y echarse en las vacas cojas. Cook (2009) indica que los colchones de superficies firmes dificultan estos movimientos de las vacas cojas ya que producen dolor en la superficie de contacto del pie al pararse, aumentando los tiempos de pie de estas vacas.

Podemos concluir que las lecherías que utilizan estabulación para sus vacas la realizan parcialmente durante la noche y durante los meses de invierno y principio de primavera. Los sistemas más utilizados son los cubículos libres, los cuales están construidos preferentemente de metal siguiendo un modelo norteamericano. Las dimensiones de estos cubículos, así como el tipo de cama no están de acuerdo a las recomendaciones de la literatura especializada, especialmente en lo que se refiere a los espacios y camas que se les ofrece a las vacas para descansar. Por las implicancias que tienen las construcciones y el diseño de los cubículos en los tiempos de descanso de las vacas y su posterior efecto en la producción de leche, presentación de patologías podales y bienestar de ellas, es necesaria una adecuada difusión a los dueños y asesores de lecherías, acerca de la importancia de realizar un adecuado diseño de estas instalaciones.

\section{RESUMEN}

Cubículos no adecuados reducen el tiempo de reposo y son un riesgo de cojeras para la vaca. En Chile existe poca información respecto a las dimensiones y camas de los cubículos. El propósito del estudio fue determinar las características de cubículos y camas usadas en lecherías del sur de Chile. Se visitaron 50 lecherías con más de 100 vacas en lactancia. Se registraron las dimensiones y tipo de cama de 1068 (10\%) de los cubículos disponibles. Los antecedentes fueron introducidos en una planilla EXCEL y se analizaron utilizando estadística descriptiva; los resultados se presentan como cuadros en base a porcentajes, promedios, rangos y medianas. El $84 \%$ (42) de las lecherías evaluadas estabulaban cinco meses durante el invierno. Un 45,5\% (29) tenían más de un cubículo/ vaca y un 34\% (15) menos de un cubículo/vaca. La combinación cubículo/ cama más usada fue cubículo de metal con colchón relleno con caucho/ paja o cama de tierra y cubículo de madera/cama de tierra. Más del $70 \%$ de los cubículos tuvo dimensiones bajo el valor referencial, para: largo del cubículo, alto al riel del cuello, alto al riel divisorio superior y distancia del muro al riel del cuello. Un $45 \%$ tuvo valores inferiores a los recomendados para el riel divisorio inferior. La altura de la solera y del posicionador del pecho, fueron excesivas en más de un $20 \%$ de los cubículos. Las lecherías utilizaban principalmente estabulación nocturna en invierno y comienzo de primavera. Los cubículos más utilizados eran metálicos, modelo norteamericano. Sus dimensiones, así como las camas, no cumplían las recomendaciones de la literatura.

\section{REFERENCIAS}

Balocchi O, R Pulido, J Fernández. 2002. Comportamiento de vacas lecheras en pastoreo con y sin suplementación de concentrado. Agri Téc 62, 87-98.

Bernardi F, J Fregonesi, C Winckler, DM Veira, MAG von Keyserlingk, DM Weary. 2009. The stall-design paradox: Neck rails increase lameness but improve udder and stall hygiene. J Dairy Sci 92, 3074-3080.
Blowey R, P Edmondson. 2000. The environment and mastitis. In Practice 22, 382-394.

Callejo A, V Jimeno. 1998. Alojamientos para vacas lecheras. Bases de producción animal. Monografía II. Alojamiento e Instalaciones. II Editorial Mundi-Prensa, Madrid, España.

Ceballos A, D Sanderson, J Rushen, DM Weary. 2004. Improving stall design: Use of 3-d kinematics to measure space use by dairy cows when lying down. J Dairy Sci 87, 2042-2050.

Chaplin SJ, HE Tement, JE Offer, DN Logue, CH Knight. 2000. A comparison of hoof lesions and behaviour in pregnant and early lactation heifers at housing. Vet $J 159,147-153$.

Cook NB, KV Nordlund. 2009. The influence of the environment on dairy cow behaviour, claw health and herd lameness dynamics. Vet $J$ 179, 360-369.

Cook NB. 2009. Free-stall design for maximum cow comfort. WCDS Advances in Dairy Technology 21, 255-268.

Drissler M, M Gaworski, CB Tucker, DM Weary. 2005 Freestall maintenance: Effects on lying behaviour of dairy cattle. J Dairy Sci 88, 2381-2387.

Ernst S, Rosenfeld C, Matamala L, Kruze J, Green L y N Tadich 2000. Antecedentes sobre el manejo de las vacas, tamaño y estructura de 523 rebaños lecheros en el sur de Chile. XXV Congreso Anual de la Sociedad Chilena de Producción Animal (SOCHIPA). Puerto Natales, Chile.

Espejo LA, MI Endres 2007 Herd-level risk factors for lameness in high-producing holstein cows housed in freestall barns. J Dairy Sci 90, 306-314.

Faull WB, J Hughes. 1985. Mastitis notes for the dairy practitioner. $3^{\text {rd }}$ ed. Liverpool University Press, Liverpool, UK.

Faull WB, J Hughes, MJ Clarkson, DY Downham, FJ Manson, JB Merritt, RD Murray, WB Russell, JE Sutherst, WR Ward. 1996. Epidemiology of lameness in dairy cattle: the influence of cubicles and indoor and outdoor walking surfaces. Vet Rec 139, 130-6.

Flower FC, DM Weary. 2006. Effect of hoof pathologies on subjective assessments of dairy cow gait. J Dairy Sci 89, 139-146.

Fulwider WK, RW Palmer. 2004. Stall usage differences of thirteen different free-stall base types. Prof Anim Sci 20,470-482.

Hughes J. 2000. Cows and cubicles. In Practice 22, 231-237.

Irish WW, RO Martin. 1983. Design considerations for free stalls. In: $2^{\text {nd }}$ Proc. Dairy Housing II, Natl. Dairy Housing Conf. Am. Soc. Agric. Eng., St. Joseph, MI. Pp 108-121.

Leonard FC, JM O'Connell, KJ O'Farrell. 1994. Effect of different housing conditions on behaviour and foot lesions in Friesian heifers. Vet Rec 134, 490-494.

Leonard FC, JM O'Connell, KJ O'Farrell. 1996. Effect of overcrowding on claw health in first calved Friesian heifers. Br Vet $J$ 152, 459-472.

McFarland DF, MJ Gamroth. 1994. Free stall designs with cow comfort in mind. In: Dairy systems for the $21^{\text {st }}$ Century, Proceedings $3^{\text {rd }}$ International Dairy Housing Conference, Pp 145-158.

McFarland DF. 2000. Free stall trouble shooting and management. In: Dairy Housing and Equipment Systems, NRAES-129. Ithaca, NY, National Resource, Agriculture and Engineering Service, Pp 247-262.

Nordlund N, NB Cook. 2003. A flowchart for evaluating dairy cow freestalls. Preconvention Seminar 7: Dairy Herd Problem Investigation Strategies. American Association of Bovine Practitioners, 36th Annual Conference, September 15-17. Columbus, OH.

Palmer R. 2005. Cow comfort issues in free stall barns. Proceedings of the $7^{\text {th }}$ Western Dairy Management Conference, March 9-11, Reno, NV, Pp 141-156.

Radostits O, K Leslie, J Fetrow. 1994. Herd health food animal production medicine. $2^{\text {nd }}$ ed. WB Saunders, Philadelphia, USA, Pp 301-303.

Tadich N, V Fuentealba, S Ernst, L Green. 1998. Información general del manejo de vacas de lechería en la X Región, Chile. Suplemento Especial. Arch Med Vet 30, 47-48.

Tadich N, N Ormeño. 2004. Factores a considerar en la estabulación de vacas lecheras Parte II. Vetermas 3, Pp 8-15. 
Tucker CB, DM Weary, D Fraser. 2005. Influence of neck-rail placement on free-stall preference, use, and cleanliness. J Dairy Sci 88, 2730-2737.

Veissier I, J Capdeville, E Delval. 2004. Cubicle design systems for cattle: comfort of dairy cows depends on cubicle adjustment. J Anim Sci, 82, 3321-3337.

Vokey FJ, CL Guard, HN Erb, DM Galton. 2001. Effects of alley and stall surfaces on indices of claw and leg health in dairy cattle housed in a free-stall barn. J Dairy Sci 84, 2686-2699.

Von Keyserlingk M, D Weary. 2009. Improving the welfare of dairy cattle: Implications of free stall housing on behaviour and health. Western Dairy Management Conference, Nevada, USA.
Wathes CM, DR Charles. 1994. Livestock Housing. CAB International. Oxon, UK.

Webster AJF. 2002. Effect of housing practices on the development of foot lesions in dairy heifers in early lactation. Vet Rec 131, 9-12.

Weschsler B, J Schaub, K Friedli, R Hauser. 2000. Behaviour and leg injuries in dairy cows kept in cubicle systems with straw bedding or soft lying mats. Appl Animal Behaviour Sci 69, 189-197.

Wierenga HK, H Hopster. 1990. The significance of cubicles for the behaviour of dairy cows. Appl Animal Behaviour Sci 26, 309-337. 